\title{
Characterization of Wavelength Interleaving in Radio-over-Fiber Systems Employing WDM/SCM
}

\author{
A. Kaszubowska, L.P. Barry, P. Anandarajah and Ling Hu \\ Research Institute in Networks and Communications Engineering, \\ School of Electronic Engineering, Dublin City University, Dublin 9, Ireland. \\ Phone: + 3531700 5883, Fax: + 3531700 5508, Email: kaszubow@eeng.dcu.ie
}

\begin{abstract}
A Radio-over-Fiber (RoF) distribution system incorporating both SCM and WDM technologies is presented. This signal is directly modulated onto three high-speed lasers. Bragg filters are employed at the receiver base station in order to both demultiplex the required optical channel, and ensure that the detected signal is single side band (in order to overcome dispersion limitations of the link). System spectral efficiency is optimised by wavelength interleaving. The channel spacing between the WDM channels is varied and the system performance for different values of channel spacing and spectral efficiencies is investigated. The results show that wavelength interleaving is a reliable technique that could be used to increase the spectral efficiency of RoF systems.
\end{abstract}

Key words: RoF system, Sub-Carrier Multiplexing, Wavelength Division Multiplexing, Wavelength Interleaving. 


\section{Introduction}

Future RoF systems could be the answer to many urgent needs of the telecommunication networks, as they could provide the necessary bandwidth for the transmission of broadband data to end-users. These systems are likely to employ two very efficient multiplexing techniques namely Sub-Carrier Multiplexing (SCM) [1] and Wavelength Division Multiplexing (WDM) [2]. This would allow many Base Stations (BS) in the system to be fed using a single fiber. Each BS would be assigned its own wavelength and signals sent to different users serviced by a particular BS would be transmitted on that wavelength by means of SCM [3-5].

In order to reduce the cost of system deployment the high frequency radio carrier (tens of gigahertz) would be generated in a Central Station (CS) and then transmitted to a BS via optical fiber. This scenario brings about two problems one of which is dispersion caused fading that occurs when high frequency signals travel along fiber [6]. This is caused by the fact that the intensity modulation of light normally generates two main side bands equi-distant from the optical carrier by the radio frequency (Double Side Band -DSB). These components are affected by chromatic dispersion, which introduces a phase shift between them. If this phase shift equals $180^{\circ}$, the two side bands will interfere destructively in the photodiode causing the output signal to fade. Dispersion caused fading could be overcome by converting the DSB signal into a Single Side Band (SSB) format [7]. The other problem entails inefficient spectral utilisation when using the standard channel spacing between WDM signals (spacing higher than twice the highest modulating frequency). There would be a large portion of unused spectrum between each wavelength and the data signal that this wavelength carries, because the radio carrier is much higher than the bandwidth occupied by the transmitted data. In order to improve the spectral efficiency of such a RoF system, a fairly new channel spacing technique called Wavelength 
Interleaving (WI) has been proposed [7]. In systems employing WI the channel spacing is reduced to values that are less than twice the highest modulating frequency. There are two possible configurations for WI (Figure 1). Scheme 1 (channel spacing larger than the Radio Frequency (RF) carrier): - demultiplexing can be achieved using a simple optical band-pass filter. In this scenario the side band of the neighbouring channel, which passes through the filter, will beat with the optical carrier producing an RF component at different frequency than the desired signal. This component could be easily removed by electrical filtration. Scheme 2 (channel spacing smaller than the RF carrier frequency) - yields better spectral efficiency, but results in higher cost because it requires either two filters in cascade or a specially designed filter with two pass band frequencies [7,8]. It is worth noting that it is possible to realise the demultiplexing and SSB conversion simultaneously using the same optical filter (see Figure 1) in both of the illustrated schemes.

In this paper we directly modulate three high-speed laser diodes with five SCM channels. The SCM channels are centred at $18.9 \mathrm{GHz}$ with the channel spacing of $450 \mathrm{MHz}$. The initial spacing between the WDM channels is set to $50 \mathrm{GHz}$, such that there is no wavelength interleaving. The demultiplexing and the SSB conversion of the desired WDM signal is performed at the receiver using an optical band-pass filter [9]. The WDM channel spacing is then reduced gradually to achieve WI (as presented in scheme 1 Figure 1) and the impact of this reduction on the system performance is verified. Both experimental and simulation results were obtained for different values of channel spacing in order to determine the factors limiting the channel spacing that could be used in WI RoF systems.

\section{Experimental set-up.}


The experimental set-up used is shown in Figure 2. A 155 Mbit/s Non-Return-to-Zero (NRZ) data stream from an Anritsu pattern generator is initially passed through a $\underline{117 \mathrm{MHz} \text { Rise Time }}$ Filter to minimize the bandwidth of the data signal. The resulting signal together five RF carriers centered at $18.9 \mathrm{GHz}$ are then applied to a mixer, which generates five Binary Phase Shift Keyed (BPSK) data signals. The SCM signal is subsequently split into three ways and used to directly modulate three high-speed Distributed Feed-Back (DFB) laser diodes from NTT Electronics. The lasers used are multiple-quantum-well devices with threshold currents around $25 \mathrm{~mA}$ and $3 \mathrm{~dB}$ electrical bandwidths in excess of $18 \mathrm{GHz}$. Specific wavelengths could be chosen due to the tunablility of the laser mode that could be achieved by temperature controlling the diode. The operating wavelengths chosen for the three lasers are 1550.3, 1550.7 and $1551.1 \mathrm{~nm}$. With each of the three lasers biased around $60 \mathrm{~mA}$, the SCM signal is applied to each of the transmitters. The optical outputs are then combined using an optical coupler and transmitted over $3 \mathrm{~km}$ of a Standard Single Mode Fiber (SSMF). The demultiplexing of the central WDM channel was performed with the aid of a Bragg Grating in conjunction with an optical circulator. The Bragg filters used are designed specifically for WDM systems with $50 \mathrm{GHz}$ spacing, and have reflection bandwidths of around $0.35 \mathrm{~nm}$. The same filter also converts the signal from a DSB to an SSB format. In order to select out only one carrier and one side-band of the central optical channel, the Bragg filter is positioned such that the central carrier is at the longest wavelength that is correctly reflected by the Bragg filter. The Bragg filter having a very sharp cut-off ensures that only one-side band is reflected. After detection the central SCM channel was downconverted by mixing the output of the photodiode with an $18.9 \mathrm{GHz}$ signal from a local oscillator. The BER of the downconverted signal was measured using an Anritsu error analyser.

\section{Experimental results.}


The BER measurements were performed for different values of optical channel spacing and the results obtained are shown in Figure 3. From the plot it can be seen that there are two sets of values for the channel spacing at which the BER remains low: above $0.33 \mathrm{~nm}$ and between $0.2-$ $0.26 \mathrm{~nm}$. The first set corresponds to conventional values of channel spacing (no WI) while the second matches scheme 1 of WI (Figure 1). The increase in Bit Error Rate (BER) for spacing smaller than $0.2 \mathrm{~nm}$ is caused by the imperfect profile of the filter used for demultiplexing (nonefficient roll-off). It can also be seen that for spacing between $0.26-0.33 \mathrm{~nm}$ the side-bands of the neighbouring WDM channels overlap, thus resulting in poor BER. The optical spectra of the WDM signal (channel spacing $0.4 \mathrm{~nm}$ ) and the demultiplexed central channel (channel spacing of $0.25 \mathrm{~nm}$ ) are shown in Figure 4 and Figure 5 respectively.

Simulations of our optical SCM/WDM system test-bed were then performed using VPI software in order to verify the factors limiting the minimum channel spacing that can be used in RoF systems employing WI. Five optical channels each carrying five SCM signals (with centre RF frequency set to $20 \mathrm{GHz}, 400 \mathrm{MHz}$ channel spacing between SCM channels and with $155 \mathrm{Mb} / \mathrm{s}$ data on each RF carrier) were combined together and sent through $10 \mathrm{~km}$ of SSMF. The central optical channel was demultiplexed and the BER for the central SCM channel was measured as a function of different WDM channel spacing $(23-50 \mathrm{GHz})$. The results are presented in Figure 6 from which it can be seen that if WI is employed the channel spacing can be reduced to a value between 25 and $38.5 \mathrm{GHz}$ to obtain a BER smaller than $10-9$ with a received optical power of -3 $\mathrm{dBm}$. The filter used had $3 \mathrm{~dB}$ bandwidth of $20 \mathrm{GHz}$ while the $20 \mathrm{~dB}$ cut-off was $37 \mathrm{GHz}$. Such a filter should allow closer placement of the WDM channels than presented in this simulation result. The factor, as regards this simulation, that ultimately limited the minimum channel spacing was the noise of the laser. As the channel spacing decreases, the SCM signal moves 
closer to the optical carrier of the adjacent channel. Reducing the channel spacing by $2 \mathrm{GHz}$ (from 25 to $23 \mathrm{GHz}$ ) increases the noise in the signal band by around $5 \mathrm{~dB}$ as can be seen in Figure 7 (optical spectrum of the demultiplexed central channel for the channel spacing of $25 \mathrm{GHz}$ ). A reduction of $3 \mathrm{GHz}$ (from 25 to $22 \mathrm{GHz}$ ) would increase the noise by $10 \mathrm{~dB}$. This noise degrades the quality of the signal, which in turn makes the channel spacing of $25 \mathrm{GHz}$ unfeasible. In order to verify the above statement, the BER vs. optical channel spacing was measured for two lasers with different linewidths. The two values chosen were 5 and $10 \mathrm{MHz}$. The optical spectra of the unmodulated laser having different linewidths are shown in Figure 8.

From the spectra one can see that when the linewidth of the laser is changed from 5 to $10 \mathrm{MHz}$ the noise level increases by around $3 \mathrm{~dB}$ at the frequency offset corresponding to the RF carrier. This has a strong impact on the system performance of the SCM/WDM system employing WI, as the Signal to Noise Ratio (SNR) would decrease with the higher noise. It could be assumed that the increase in the noise level is mainly due to higher phase noise, as that this type of noise is associated with the broadening of the laser linewidth. The obtained BER as a function of optical channel spacing for the same system configuration as described before but with the abovementioned laser linewidths are shown in Figure 9.

As predicted a system based on the $5 \mathrm{MHz}$ linewidth laser yields a better performance than that with the $10 \mathrm{MHz}$ linewidth. From Figure 9 it can also be seen that the BER remains more or less constant for the value of channel spacing between 31 and $37 \mathrm{GHz}$. As the channel spacing is reduced to values below $30 \mathrm{GHz}$ the BER curve rises much more quickly as does the phase noise of the neighbouring channel (received signal approaching optical carrier see Figure 8). In both cases the system performance degrades gradually, nevertheless the difference between the two curves is preserved. This proves that the increase in BER is primarily caused by the noise of the 
laser and not by the insufficient roll-off of the filter used for demultiplexing, as the latter would cause the neighbouring carrier to leak through. This in turn would bring about an immediate deterioration of the BER for both systems (which is shown to occur when the channel spacing is reduced to $22.5 \mathrm{GHz}$ ).

\section{Conclusions}

The successful development of future hybrid RoF systems employing both SCM and WDM technologies is highly dependent on the use of simple and efficient technologies that involve generating optical microwave signals at the central station and demultiplexing signals at the remote base stations. In this paper we have demonstrated a hybrid WDM/SCM based radio-overfiber distribution system employing the technique of WI, which improves the spectral efficiency of the system. We have shown that by using an optical band-pass filter, the optical channel spacing between the WDM channels (each carrying $20 \mathrm{GHz}$ RF signals) can be reduced to 25 GHz. This means a $40 \%$ reduction in required spectrum in comparison to the scenario when no WI is employed (minimal channel spacing of $42 \mathrm{GHz}$ is required). To characterize the technique of WI, we have also performed a series of experiments to identify factors, which set a limit on the minimum optical spacing that could be used. The results presented show that the roll-off of the filter used for demultiplexing as well as the phase noise of the transmitter act as the major limiting factors restraining the spectral efficiency that could be achieved using WI. 


\section{References}

1. O. K. Tonguz et al. "Personal Communications Access Networks Using Subcarrier Multiplexed Optical Links" J. Lightwave Technol., vol.14, (1996) 1400-1408

2. A. Stohr et al. "Full-Duplex $60 \mathrm{GHz}$ Fiber Optic Transmission" Electron. Lett., vol. 35, (1999) 1653-1655

3. G. H. Smith, D. Novak, and C. Lim, "A millimeter-wave full-duplex fiber-radio star-tree architecture incorporating WDM and SCM", IEEE Photon. Technol. Lett., vol. 10, (1998) 1650-1652

4. E. Vourch, D. Le Berre, and D. Herve, "A WDM fiber-radio experiment incorporating a wavelength tunable single-side-band filter", Proc. Microwave Symposium Digest, 2002 IEEE MTT-S International, vol. 3, (2002) 1703 -1706, Seattle, USA

5. H. Toda, T. Yamashita, K. Kitayama, and T. Kuri, "DWDM demultiplexing with $25 \mathrm{GHz}$ channel spacing for $60 \mathrm{GHz}$ band radio-on-fibre systems", Proc. European Conference on Optical Communications, vol. 3, (20025) Paper 8.2.4, Copenhagen, Denmark

6. U. Gliese, S. Norskov, T. N. Nielsen "Chromatic Dispersion in Fiber-Optic Microwave and Millimeter-Wave Links", IEEE Trans. Microwave Theory Tech., vol. 44, (1996) $1716-1724$

7. G. H. Smith, D. Novak, Z. Ahmed, "Overcoming Chromatic - Dispersion Effects in Fibre-Wireless Systems Incorporating External Modulators", IEEE Trans. Microwave Theory Tech., vol. 45, 19971410 - 1415

8. C. Marra et al. "Wavelength-Interleaved OADMs Incorporating Optimized Multiple Phase-Shifted FBRs for Fiber-Radio Systems" J. Lightwave Technol., vol. 21, (2003) 3239 
9. A. Kaszubowska, P. Anandarajah, L. P. Barry "Multifunctional operation of a fiber Bragg grating in a WDM/SCM radio over fiber distribution system", IEEE Photon. Technol. Lett., vol. 16, 2004 605- 607 


\section{Figure Captions}

Figure 1: Different schemes of WDM channel allocation in WI systems

Figure 2: Experimental set-up

Figure 3: BER vs. channel spacing

Figure 4: Optical spectrum of the WDM signal (channel spacing $0.4 \mathrm{~nm}$ )

Figure 5: Optical spectrum of the demultiplexed central cannel (channel spacing $0.25 \mathrm{~nm}$ )

Figure 6: BER vs. optical channel spacing for the central SCM channel

Figure 7: Optical spectrum of the demultiplexed central channel (channel spacing $25 \mathrm{GHz}$ )

Figure 8: Optical spectrum of the unmodulated laser with the linewidth of $5 \mathrm{MHz}$ (grey) and 10 $\mathrm{MHz}$ (black) (resolution bandwidth of $10 \mathrm{MHz}$ )

Figure 9: BER vs. optical channel spacing for two values of laser linewidth: squares $-5 \mathrm{MHz}$, diamonds $-10 \mathrm{MHz}$ linewidth 